\title{
Synthesis and Spectral-Luminescence Properties of Ytterbium Complexes with $\beta$-Diketonate Derivatives of Hematoporphyrin
}

\author{
Sergey A. Zhuravlyov, ${ }^{a}$ Gelii V. Ponomarev, ${ }^{\text {b }}$ Natalya V. Rusakova, ${ }^{a}$ and \\ Yuriy V. Korovina @
${ }^{a}$ A.V. Bogatsky Physico-Chemical Institute of National Academy of Sciences, Odessa, 65080, Ukraine
${ }^{\mathrm{b}}$ Institute of Biomedical Chemistry of Russian Academy of Medical Sciences, Moscow, 11912, Russia
@Corresponding authorE-mail: lanthachem@te.net.ua

\begin{abstract}
In this work the synthesis, optical and luminescence spectroscopic characterization of the new ytterbium complexes with asymmetric porphyrins of hematoporphyrin group, modified by two $\beta$-diketone fragments are presented. All ligands and complexes are investigated by the methods of molecular mechanics, elemental analysis, UV-vis, IR, luminescence, mass and ${ }^{1} H$ NMR spectroscopy. Influence of some factors (such as location of $\beta$-diketones substituents, value of charges on ytterbium ion, etc.) on the value of 4 -luminescence is discussed.
\end{abstract}

Keywords: Hematoporphyrin, ytterbium complex, $\beta$-diketonate derivatives, $4 f$-luminescence.

\section{Introduction}

Properties of lanthanides complexes with hematoporphyrin and its derivatives have been studied only scarcely. ${ }^{[1-3]}$ Interest to these macroheterocycles is connected first of all with possible near IR-luminescence of $\mathrm{Ln}^{\text {III }}$ complexes $(\mathrm{Ln}=$ neodymium, erbium and ytterbium $){ }^{[4]}$ Such factors, as the minimum signal from bio-objects, possibility of excitation of $4 f$-luminescence in a wide spectral range (250-650 nm), organotropy of hematoporphyrin complexes determine the perspectives of such studies. ${ }^{[5-7]}$ On the other hand, choice of ytterbium as the coordinating centre is conditioned by the fact that from of all lanthanide series only $\mathrm{Yb}$ ions show intensive $4 f$-luminescence in porphyrin complexes. ${ }^{[8]}$

In this work the synthesis and spectral-luminescence properties of ytterbium complexes with hematoporphyrin derivatives, modified asymmetrically by two lateral $\beta$-diketonates fragments, namely, diacetylacetonates and dithenoyltrifluoroacetonates (compounds $\mathbf{1}$ and 2, respectively, Scheme 1), are described.

\section{Experimental}

All reagents and solvents were of reagent-grade quality obtained from commercial suppliers. Compounds $\mathbf{1}$ and $\mathbf{2}$ were prepared according the literature method. ${ }^{[9]}$

Synthesis of complexes $\mathbf{3}$ and $\mathbf{4}$ was performed by interaction of diacetylacetonate $\mathbf{1}$ or dithenoyltrifluoroacetonate 2 of hematoporphyrin $\left(2 \cdot 10^{-4} \mathrm{~mol}\right)$ with ytterbium acetylacetonate $\left(5 \cdot 10^{-3} \mathrm{~mol} ;\right.$ acetylacetone - Acac as an extra-ligand) in 1,2,4-trichlorobenzene $(25 \mathrm{ml})$ during 4-6 hours. After the reaction completion 1,2,4-trichlorobenzene was sublimated and then crude product was precipitated with water, filtered off and separated by column chromatography on silica. Yield $76-80 \%$. It should be noted that it is very difficult to interpretate correctly the chemical shifts of protons in the ${ }^{1} \mathrm{H}$ NMR spectra of complexes $\mathbf{3}$ and $\mathbf{4}$ because of brightly expressed paramagnetic properties of ytterbium ions.
Therefore for comparison we have used the lutetium complexes with ligands $\mathbf{1}$ and $\mathbf{2}$ received analogously to ytterbium complexes and having the same structure.

$Y b($ Acac)-diacetylacetonate hematoporphyrin, 3. Yield 80\%. Found: $\mathrm{C} 57.0, \mathrm{H} 5.9, \mathrm{Yb} 15.9 \% . \mathrm{C}_{51} \mathrm{H}_{61} \mathrm{~N}_{4} \mathrm{O}_{10} \mathrm{Yb}$ requires $\mathrm{C} 57.6$, $\mathrm{H} 5.8, \mathrm{Yb} 16.3$ (\%). $\mathrm{m} / z$ (FAB) \%: 1064 (92) [(M-YbAcac) $\left.{ }^{+}\right]$. UVvis $\lambda_{\text {max }}$ (DMF) nm (lge): 414.6 (4.6), 540.5 (3.5), 575.2 (3.6).

Lu(Acac)-diacetylacetonate hematoporphyrin, 3a. Yield 79\%. Found: $\mathrm{C} 57.3, \mathrm{H} 5.8, \mathrm{Lu} 16.2 \%$. $\mathrm{C}_{51} \mathrm{H}_{61} \mathrm{~N}_{4} \mathrm{O}_{10} \mathrm{Lu}$ requires $\mathrm{C}$ 57.5, H 5.8, Lu 16.4 \%. m/z (FAB) \%: 1064 (96) [(M-LuAcac) $\left.)^{+}\right] .{ }^{1} \mathrm{H}$ NMR ([D $]$ DMSO, $300 \mathrm{~K}) \delta_{\mathrm{H}}$ ppm: $-2.73(2 \mathrm{H}$, s, pyrrole-NH), 10.110.28 (4H, s, meso-H), 3.6-3.65 (12H, m, $\mathrm{CH}_{3}$-ring), 4.38-4.46 (4H, q, $\left.\alpha-\mathrm{CH}_{2}\right), 3.24-3.35$ (4H, q, $\left.\beta-\mathrm{CH}_{2}\right), 2.24\left(12 \mathrm{H}, \mathrm{s}, \mathrm{CH}_{3}\right.$-Acac).

$\mathrm{Yb}$ (Acac)-dithenoyltrifluoroacetone hematoporhyrin, 4. Yield 76\%. Found: C 52.9, H 4.2, $\mathrm{Yb} 13.0 \%$. $\mathrm{C}_{57} \mathrm{H}_{55} \mathrm{~N}_{4} \mathrm{O}_{10} \mathrm{~F}_{6} \mathrm{~S}_{2} \mathrm{Yb}$ requires C 52.4, H 4.2, Yb 13.2 \%. m/z (FAB) \%: 1307 (90) [(M-YbAcac) ${ }^{+}$]. UV-vis $\lambda_{\text {max }}$ (DMF) nm (lge): 403.9 (4.2), 540.7 (3.1), 579.9 (3.2).

Lu(Acac)-dithenoyltrifluoroacetone hematoporhyrin, 4a. Yield 77\%. Found: C 52.4, H 4.3, Lu 13.2\%. $\mathrm{C}_{57} \mathrm{H}_{55} \mathrm{~N}_{4} \mathrm{O}_{10} \mathrm{~F}_{6} \mathrm{~S}_{2} \mathrm{Lu}$ requires C 52.3, H 4.2, Lu $13.4 \%$. m/z (FAB) \%: 1308 (93) [(M-Lu Acac $\left.)^{+}\right] .{ }^{1} \mathrm{H}$ NMR $\left(\left[D_{6}\right] \mathrm{DMSO}, 300 \mathrm{~K}\right) \delta_{\mathrm{H}} \mathrm{ppm}:-2.7(2 \mathrm{H}$, s, pyrrole$\mathrm{NH}), 10.11-10.29$ (4H, s, meso-H), 3.64-3.65 (12H, m, $\mathrm{CH}_{3}$-ring), 4.07-4.11 (4H, q, $\left.\alpha-\mathrm{CH}_{2}\right), 3.24-3.35\left(4 \mathrm{H}, \mathrm{q}, \beta-\mathrm{CH}_{2}\right), 7.17(2 \mathrm{H}, \mathrm{m}$, 4-thenoyl), 7.92 (2H, m, 5-thenoyl), 7.97 (2H, m, 2-thenoyl).

All ligands and complexes were isolated in solid state and characterized by elemental analysis, IR, UV-vis, ${ }^{1} \mathrm{H}$ NMR and FAB MS spectra.

Elemental analysis was performed on Perkin-Elmer Model 240C elemental analyzer. Mass spectra FAB were recorded on Varian MAT CH-112 spectrometer (Varian BV, Middelburg) using $m$-nitrobenzyl alcohol as the matrix. ${ }^{1} \mathrm{H}$ NMR spectra were recorded on Bruker Avance-300 (300 MHz) in $\mathrm{CDCl}_{3}$ and DMSO.

Absorption spectra were recorded on Specord M40 UV/ VIS (Carl Zeiss, Jena, Germany) and Lambda 9 UV/VIS/NIR (Perkin-Elmer) spectrometers. Luminescence measurements were carried out on SDL-2 spectrofluorimeter equipped with a photon counting system (Optic-Mechanical Association, St.-Petersburg, Russia). All luminescence spectra were corrected with a standard lamp. A xenon lamp (Xe-150) was used as the excitation source. The solutions of complexes $(1 \mathrm{ml})$ were placed into $10-\mathrm{mm}$ quartz cuvette. The emission of the solutions was recorded at the angle 


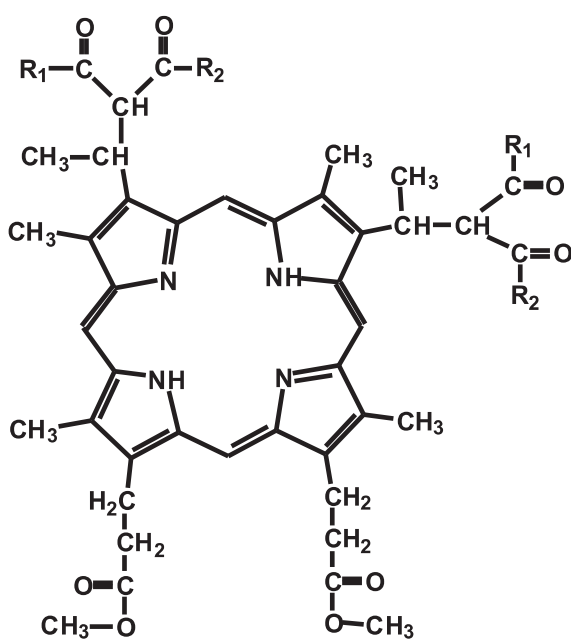

1,2

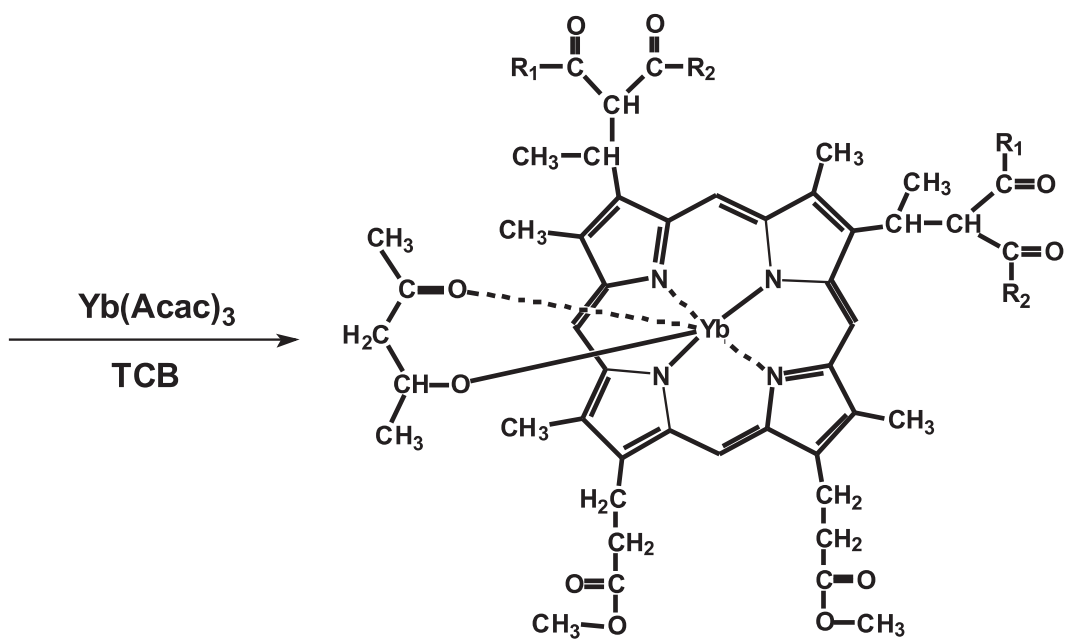

3,4

\section{1,3: $\quad \mathbf{R}_{1}=\mathbf{R}_{2}=\mathrm{CH}_{3}$ \\ 2,4: $\quad R_{1}=C_{3}, R_{2}=C_{4} H_{3} S$}

Scheme 1. Synthesis of $\mathrm{Yb}$ complexes 3 and $\mathbf{4}$.

of $90^{\circ}$ to the exciting radiation. The position of ligand triplet (T) levels was obtained from phosphorescence spectra of ligands in complexes with lutetium at $77 \mathrm{~K}$. The relative quantum yields of $4 f$-luminescence $(\varphi)$ for the ytterbium complexes were calculated by the method described before ${ }^{[8]}$ using Zn-tetraphenylporphyrin as a standard $(\varphi=0.0315$ in ethanol). All luminescent measurements were carried out at $25^{\circ} \mathrm{C}$ in DMF.

\section{Results and Discussions}

In addition to elemental analysis, the porphyrin nature of these reagents is reliably described by the IR spectra. The band at $1734 \mathrm{~cm}^{-1}\left(v_{\mathrm{C}=\mathrm{O}}\right)$ in all of these substances, which is absent in the IR-spectrum of tetramethyl ester of hematoporphyrin (TMHP), indicates the presence of $\beta$-diketonate fragments. The difference in the spectra of ytterbium complexes and free-base porphyrins is expressed by the absence of intensive band of N-H vibrations $\left(980 \mathrm{~cm}^{-1}\right)$. Individual bands at 773 and $826 \mathrm{~cm}^{-1}$ in the case of the free base porphyrin were converted into the weak shoulders in the region of $800 \mathrm{~cm}^{-1}$. The band in the region of 470-570 $\mathrm{cm}^{-1}$ corresponds to the skeletal vibrations of $\mathrm{Yb}-\mathrm{N}$ bond in the porphyrin macrocycle.

It should be noted that the absence of considerable changes of chemical shifts of peripheral group protons confirms the fact that no complexation on peripheral $\beta$-diketonate fragments takes place. To illustrate this conclusion we cite below the data of ${ }^{1} \mathrm{H}$ NMR spectra of the free base $\beta$-diketonates hematoporphyrin derivatives: diacetylacetonate hematoporphyrin $\mathbf{1}-\delta_{\mathrm{H}}\left(\left[D_{6}\right] \mathrm{DMSO}, 300\right.$ K) -3.77 (2H, s, pyrrole-NH), 10.1-10.3 (4H, s, meso-H), 3.59-3.67 (12H, m, $\mathrm{CH}_{3}$-ring), 4.38-4.46 (4H, q, $\left.\alpha-\mathrm{CH}_{2}\right)$, 3.24-3.35 (4H, q, $\left.\beta-\mathrm{CH}_{2}\right), 2.31\left(12 \mathrm{H}, \mathrm{s}, \mathrm{CH}_{3}\right.$-Acac); dithenoyltrifluoroacetone hematoporhyrin $2-\delta_{\mathrm{H}}\left(\left[D_{6}\right]\right.$ DMSO, $300 \mathrm{~K})-3.74$ (2H, s, pyrrole-NH), 10.11-10.3 (4H, s, meso-H), 3.64-3.7 (12H, m, $\mathrm{CH}_{3}$-ring), 4.07-4.14 (4H, q, $\left.\alpha-\mathrm{CH}_{2}\right), 3.29-3.33\left(4 \mathrm{H}, \mathrm{q}, \beta-\mathrm{CH}_{2}\right), 7.3$ (2H, m, 4-thenoyl), 8.0 (2H, m, 5-thenoyl), 8.2 (2H, m, 2-thenoyl).

The absorption spectra of the free-base porphyrins contain the Soret band, which exhibits insignificant changes when introducing different $\beta$-diketonates fragments, and four $Q$-bands in the visual region, typical for porphyrins (Table $1)$. In the spectra of complexes the Soret band is shifted bathochromically $(\Delta \lambda \approx 2-12 \mathrm{~nm})$ with intensity decreasing. In addition four $Q$-bands are degenerated into two bands displaced hypsochromically at 540 and $575 \mathrm{~nm}$. This fact demonstrates the change of macrocycle symmetry and serves as one of the proofs of complexation process.

Thus, the obtained data prove the formation of Ln complexes of ratio Ln:ligand $=1: 1$. Moreover, the complexation takes place in the cavity of porphyrin macrocycle but not on peripheral $\beta$-diketonate fragments.

The spectra of molecular luminescence for $\mathbf{1}$ and $\mathbf{2}$ are similar and consist of two bands in the region of 610-700 nm $\left(\lambda_{1}=619 \mathrm{~nm}, \lambda_{2}=678 \mathrm{~nm}(1) ; \lambda_{1}=623 \mathrm{~nm}, \lambda_{2}=680 \mathrm{~nm}\right.$ (2)). A weak band in the region of $635-643 \mathrm{~nm}$ is conditioned by two processes, namely, formation of photoproducts from the aggregates of porphyrins and photodestruction of compounds. All ytterbium complexes exhibit $4 f$-luminescence of $\mathrm{Yb}^{\mathrm{III}}$ ions in the region of 960-1010 nm $\left(\lambda_{\text {max }}=980 \mathrm{~nm},{ }^{2} F_{5 / 2} \rightarrow{ }^{2} F_{7 / 2}\right.$ transition) at excitation in a wide spectral range $(250-650 \mathrm{~nm})$. The almost full coincidence of the excitation and absorption spectra of $\mathrm{Yb}$-complexes indicates that the excitation energy is transferred from the $T$-levels of porphyrin moiety $\left(E_{\mathrm{T}}=\right.$ $14750 \mathrm{~cm}^{-1}$ and $E_{\mathrm{T}}=13900 \mathrm{~cm}^{-1}$ for complexes 3 and $\mathbf{4}$, respectively) to the radiative ${ }^{2} F_{7 / 2}$-level $\left(E_{\mathrm{Yb}}{ }^{3+}=10200 \mathrm{~cm}^{-1}\right)$ of ytterbium ions due to the intramolecular mechanism of transfer. In complexes $\mathbf{3}$ and $\mathbf{4}$ the molecular luminescence is practically absent which confirms the effectiveness of this process.

It is known that $4 f$-luminescence in the near IR spectral range essentially depends on the value of energy gap $\left(\Delta E=E_{\mathrm{T}}\right.$ $\left.-E_{\mathrm{Ln}}{ }^{\mathrm{III}}\right){ }^{[10]}$ Comparison of this parameter for diacetylacetonate and dithenoyltrifluoroacetonate derivatives $(\Delta E=4550$ and $3700 \mathrm{~cm}^{-1}$, respectively) allows to explain the lesser value of nonradiative losses and increasing of quantum yield of complex 4 in 3.8 times relative to 3 .

Some factors which influence on efficiency of luminescence signal from $\mathrm{Yb}$-complexes were considered. 
Synthesis and Spectral-Luminescence Properties of Ytterbium Complexes

Table 1. Spectral-luminescence properties of compounds studied (solutions in DMF).

\begin{tabular}{|c|c|c|c|c|c|c|}
\hline \multirow{3}{*}{ Compounds } & \multicolumn{5}{|c|}{$\lambda_{\max }(\lg \varepsilon), n m$} & \multirow{3}{*}{$\varphi \cdot 10^{3 \mathrm{c}}$} \\
\hline & \multirow{2}{*}{ Soret band } & \multicolumn{4}{|c|}{$Q$-bands } & \\
\hline & & IV & III & II & I & \\
\hline 1 & $402.8(5.4)$ & $500.5(4.2)$ & $534.7(4.0)$ & 570.7 (3.9) & 622.5 (3.6) & \\
\hline 2 & $401.7(5.4)$ & $500.5(4.1)$ & $534.7(4.0)$ & 568.8 (3.9) & 622.6 (3.7) & \\
\hline 3 & $414.6(4.6)$ & & & $540.5(3.5)$ & $575.2(3.6)$ & 1.2 \\
\hline 4 & $403.9(4.2)$ & & & $540.7(3.1)$ & $572.9(3.2)$ & 4.6 \\
\hline TMHPa & $401.4(5.2)$ & $500.5(4.1)$ & 533.6 (3.9) & 568.8 (3.8) & $622.6(3.6)$ & \\
\hline Yb-TMHP & $402.4(4.3)$ & & & $540.4(3.4)$ & $579.8(3.4)$ & 0.6 \\
\hline
\end{tabular}

${ }^{\mathrm{a}}$ and ${ }^{\mathrm{b}}$ are presented for comparison; ${ }^{\mathrm{c}}$ - excitation in the Soret band maximum.

Table 2. Atom charges and bond lengths of $\beta$-diketone derivatives of hematoporphyrin.

\begin{tabular}{|c|c|c|c|c|c|c|c|}
\hline \multirow{2}{*}{ Compounds } & \multirow{2}{*}{$\begin{array}{l}\text { Charge on } \\
\text { Yb, relative } \\
\text { charge units } \\
\text { (c.u.) }\end{array}$} & \multicolumn{6}{|c|}{ Atom charges, c.u. / Yb-X bond length, $\AA$} \\
\hline & & $\mathrm{N}_{1}$ & $\mathrm{~N}_{2}$ & $\mathrm{~N}_{3}$ & $\mathrm{~N}_{4}$ & $\mathrm{O}(=)$ & $\mathrm{O}(-)$ \\
\hline TMHP & \multirow{3}{*}{1.6573502} & -0.1882128 & -0.1656210 & -0.1655682 & -0.1882619 & & \\
\hline \multirow{2}{*}{ Yb-TMHP } & & $-0.4271759 /$ & $-0.4271281 /$ & $-0.4271762 /$ & $-0.4271283 /$ & $-0.1226794 /$ & $-0.0348687 /$ \\
\hline & & 2.232 & 2.234 & 2.236 & 2.233 & 2.185 & 2.228 \\
\hline 1 & & -0.1882575 & -0.1656227 & -0.1656164 & -0.1882622 & & \\
\hline 2 & & -0.1882557 & -0.1656210 & -0.1656147 & -0.1882625 & & \\
\hline \multirow{2}{*}{3} & \multirow{2}{*}{1.6573583} & $-0.4271340 /$ & $-0.4271277 /$ & $-0.4271334 /$ & $-0.4271277 /$ & $-0.1226796 /$ & $-0.034869 /$ \\
\hline & & 2.229 & 2.234 & 2.227 & 2.229 & 2.188 & 2.225 \\
\hline \multirow{2}{*}{4} & \multirow{2}{*}{1.6573517} & $-0.4271340 /$ & $-0.4271277 /$ & $-0.4271334 /$ & $-0.4271277 /$ & $-0.1226796 /$ & $-0.034869 /$ \\
\hline & & 2.230 & 2.228 & 2.230 & 2.226 & 2.188 & 2.222 \\
\hline
\end{tabular}

In particular, the observable difference in values of $4 f$-luminescence quantum yield can depend on change of partial charges on nitrogen donor atoms in porphyrin macrocycle, on polarity of the $\mathrm{Yb}-\mathrm{N}$ bond, and on stereo electronic effects of $\beta$-diketone substituents. We used the model "structure-quantum yield of $4 f$-luminescence" for consideration of these factors. Molecular mechanics simulations were performed by two different molecular modelling programs $\left(\mathrm{ELZA}^{[11]}\right.$ and HyperChem $\left.{ }^{[12]}\right)$. The structural characteristics of $\beta$-diketone substituents were considered as the structural descriptors in these models. It was established (by ELZA-method) that insignificant reduction of charge on nitrogen atoms of $\mathbf{3}$ and $\mathbf{4}$ (in comparison to $\mathrm{Yb}$-TMHP) and reduction of charge on metal atom takes place (Table 2). Obviously, the bond lengths between ytterbium and nitrogen atoms are unequal unlike symmetrical porphyrins. Since the $\beta$-diketone substituents in porphyrin molecule are rather far from the coordination centre it is not possible to interpret unequivocally their electronic specificity.

The geometry of the investigated molecules is directly considered by calculations with a help of program HyperChem. It is exposed that presence of $\beta$-diketone substituents leads to the small increase of coordination cavity diameter of both porphyrins and their ytterbium complexes: $4.34 \AA$ (3) and $4.47 \AA$ (4) in comparison to Yb-TMHP (4.32 $\AA$ ). It is necessary to take into account also the presence of extra- ligand (acetylacetonate in this case). It is known ${ }^{[13]}$ that this extra-ligand as the complexation agent provokes some withdrawing of ytterbium ion from the macrocycle plane on $0.57 \AA$ (3) and $0.51 \AA$ (4), what is less on $0.07 \div 0.13 \AA$ than in Yb-TMHP. The obtained data correlate in a good way with increase of $4 f$-luminescence quantum yield (Table 1). We've calculated that the spatial structure of complexes received by molecular mechanics geometry optimisation corresponds to distorted tetrahedral pyramid (Figure 1).

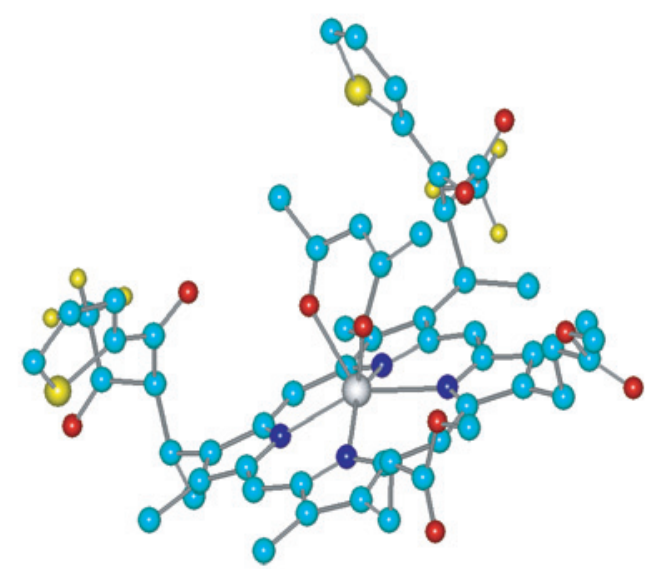

Figure 1. Structure of $\mathbf{4}$ optimized by HyperChem (method $\mathrm{MM}^{+}$, Polak-Ribiere optimization). 


\section{Conclusions}

Spectral-luminescent behaviour of the new ytterbium complexes with asymmetric porphyrins of hematoporphyrin group was investigated. It was found that efficiency of 4 -luminescence of these complexes depends on correlation between bond lengths and diameter of the cavity of porphyrin macrocycle and, at the same time, practically doesn't depend on the values of partial charges of atoms in the coordination centre.

\section{References}

1. Gaiduk M.I., Grigoryants V.V., Mironov A.F., Roytman L.D., Chissov V.I., Rumyantseva V.D., Sukhin G.M. Doklady $A N$ SSSR 1989, 309, 980-983. (in Russ.).

2. Gaiduk M.I., Grigoryants V.V., Mironov A.F., Rumyantseva V.D., Roytman L.D. J. Applied Spectroscopy 1987, 46, 163-166.

3. Horrocks W., Venteicher R., Spilburg C. Biochem. Biophys. Res. Commun. 1975, 64, 317-322.
4. Tsvirko M.P., Stelmakh G.F., Pyatosin V.E., Solovyov K.N., Kachura T.F., Piskarskas A.S., Gadonas R.A. Chem. Phys. 1986, 106, 467-476.

5. Hong Z.R., Liang C.J., Li R.G. Thin Solid Films 2001, 391, 122-125.

6. Berenbaum M.G., Bonnett R., Scourides P.A. Brit. J. Cancer 1982, 45, 571-581.

7. Sternberg E., Dolphin D., Bruckner C. Tetrahedron 1998, 54, 4151-4202.

8. Shushkevich I.K., Dvornikov S.S., Kachura T.F., Solovyov K.N. J. Applied Spectroscopy 1981, 35, 1109-1113.

9. Ponomarev G.V., Kirilova G.V., ShulgaA.M. Khim. Geterotsikl. Soedin. 1991, 11, 1564-1565. (in Russ.).

10. Parker D., Williams J.A.G. J. Chem. Soc. Perkin Trans. 2 1995, 1305-1314.

11. Kuz'min V.E., Berestetskaya E.L. Zh. Strukt. `Khim. 1983, 24, 131-132. (in Russ.)

12. HyperChem, version 5.1, Hypercube, Inc., Gainesville, FL, 1998.

13. Berezin B.D., Koifman O.I. Uspekhi Khimii 1973, 42, 20072036. (in Russ.).

Received 12.01.2009

Accepted 14.03.2009

First published on the web 15.04.2009 